\title{
The Running Coupling from the Quark-Gluon Vertex
}

\author{
Jon Ivar Skullerud ${ }^{a}$
}

${ }^{a}$ CSSM and Department of Physics and Mathematical Physics, The University of Adelaide, Adelaide, SA 5005, Australia and UKQCD Collaboration

\begin{abstract}
We present results for the QCD running coupling obtained from measuring the quark-gluon vertex in Landau gauge with suitable renormalisation conditions. The issue of discretisation errors arising from the fermion action is discussed.
\end{abstract}

\section{Introduction}

The quark-gluon vertex is one of the fundamental quantities of QCD, and studying the form of the vertex can give us greater insights into the dynamics of the theory and may provide and important necessary input into Dyson-Schwinger equations. Here we will focus on using the quarkgluon vertex to extract the running coupling from first principles.

\section{The vertex function}

The full (unamputated) momentum space vertex function can be defined as

$G_{\mu}^{a}(p, q)_{\alpha \beta}^{i j}=\left\langle S_{\alpha \beta}^{i j}(p) A_{\mu}^{a}(q)\right\rangle$

and the amputated (OPI) vertex function

$$
\begin{aligned}
& \Lambda_{\mu}^{a, \text { lat }}(p, q)= \\
& \quad\langle S(p+q)\rangle^{-1}\left\langle S(p) A_{\mu}^{a}(q)\right\rangle\langle S(p)\rangle^{-1}\langle D(q)\rangle^{-1}
\end{aligned}
$$

$D(q)$ is the scalar part of the gluon propagator, given in the Landau gauge by

$D_{\mu \nu}^{a b}(q)=\delta^{a b} T_{\mu \nu}(q) D(q)$

where $T_{\mu \nu}$ is the projection onto transverse fields.

Using the requirements of Lorentz covariance and parity and charge conjugation invariance, one can derive the following general form for the vertex in the continuum:

$$
\begin{aligned}
\Lambda_{\mu}\left(p^{2}, q^{2}, p q\right) \equiv \frac{1}{N_{C}^{2}-1} \operatorname{Tr}_{c o l} T^{a} \Lambda_{\mu}^{a}(p, q) \\
=F_{1} p_{\mu}+F_{2} q_{\mu}+F_{3} \gamma_{\mu} \\
\quad+F_{4} \not p p_{\mu}+F_{5} \not p q_{\mu}+F_{6} \not p_{\mu}+F_{7} \not q q_{\mu}
\end{aligned}
$$

$$
\begin{aligned}
& +F_{8} \sigma_{\mu \nu} p^{\nu}+F_{9} \sigma_{\mu \nu} q^{\nu} \\
& +F_{10} \epsilon_{\mu \nu \kappa \lambda} \gamma_{5} \gamma^{\nu} p^{\kappa} q^{\lambda}
\end{aligned}
$$

where all the $F$ 's depend only on the invariants $p^{2}, q^{2}$ and $p q$.

At tree level, this reduces to $\Lambda_{\mu}^{0}=\frac{i}{2} g_{0} \gamma_{\mu}$. From this we can see that the form factor containing the running coupling is $F_{3}$, while all the other form factors are expected to be finite.

If we define

$K_{\mu}(p, q) \equiv i \operatorname{Tr} \gamma_{\mu} \Lambda_{\mu}(p, q)$

we find that $i F_{3}\left(p^{2}, 0,0\right)=\left.K_{\mu}(p, 0)\right|_{p_{\mu}=0}$.

This kinematics can then be used to define a momentum subtraction scheme for the renormalised coupling:

$g_{R}^{M O M}(\mu)=-\left.2 i Z_{\psi}(\mu) Z_{A}^{1 / 2}(\mu) F_{3}\left(p^{2}, 0,0\right)\right|_{p^{2}=\mu^{2}}$

where $Z_{\psi}$ and $Z_{A}$ are the renormalisation constants for the quark and gluon fields, defined in the Landau gauge by

$$
\begin{aligned}
\left.D\left(p^{2}\right)\right|_{p^{2}=\mu^{2}} & =Z_{A}(\mu) \frac{1}{\mu^{2}} \\
\operatorname{Tr}(\gamma \tilde{p}) S^{-1}(\tilde{p}) /\left.\tilde{p}^{2}\right|_{p^{2}=\mu^{2}} & =\frac{i}{Z_{\psi}(\mu)}
\end{aligned}
$$

$\tilde{p}_{\mu}=\frac{1}{a} \sin k_{\mu} a$ is used in the definition of $Z_{\psi}$ to make it more 'continuum-like'.

$g_{R}^{M O M}$, as defined in (6) can then be related perturbatively to the running coupling calculated in other schemes, eg. $g_{R}^{\overline{M S}}\left(q^{2}\right)$. This matching can be performed entirely within continuum perturbation theory [1]. 
The running of $g_{R}^{M O M}$ can also be compared to the perturbative continuum coupling derived from the two-loop beta function

$g^{2}(\mu)=\left[b_{0} \ln \left(\mu^{2} / \Lambda^{2}\right)+\frac{b_{1}}{b_{0}} \ln \ln \left(\mu^{2} / \Lambda^{2}\right)\right]^{-1}$

with $b_{0}=11 / 16 \pi^{2}, b_{1}=102 /\left(16 \pi^{2}\right)^{2}$, by doing a one-parameter fit to this formula.

\section{Computation and results}

332 quenched configurations have been analysed at $\beta=6.0\left(a^{-1}=2 \mathrm{GeV}\right)$ with a lattice size of $16^{3} \times 48$. The propagators have been generated using the tadpole improved Sheikholeslami-Wohlert action, at $\kappa=0.137$. The gauge fields and propagators have been fixed to Landau gauge, with accuracy $\frac{1}{V N_{c}} \sum_{x, \mu}\left|\partial_{\mu} A_{\mu}(x)\right|^{2}<10^{-12}$.

The full vertex $\gamma_{\mu} G_{\mu}(p, q)$ has a clear, symmetric signal for $q=0$ and all values of $p$ where $p_{\mu}=0$ [2]. For $q \neq 0$ the signal falls off rapidly, and disappears entirely for $|q a| \geq \frac{\pi}{4}$. This makes it difficult to implement a renormalisation scheme where $q \neq 0$, although this would be preferable.

In order to compute the proper vertex, one needs the quark renormalisation constant $Z_{\psi}$. As figure 1 shows, this suffers from serious ambiguities, especially at high momenta. A more detailed analysis shows these ambiguities to be a result of violation of rotational symmetry, which can be attributed to $O(a)$ errors in the fermion action: although the SW action is $O(a)$ improved, this is only the case for on-shell, gauge invariant quantities. In order to remove $O(a)$ errors for gauge dependent, off-shell quantities, one requires two additional counterterms in the actions [3].

\subsection{The proper vertex and $g_{R}$}

One feature of the data is the strong correlations between data at different momenta. This makes an assessment of possible violations of rotational and reflection symmetry difficult, as it turns out that different samples of 83 configurations can have values of $K_{\mu}$ as much as $3 \sigma$ apart. With this proviso, we find that for $q=0$ and $p_{\mu}=0, K_{\mu}(p, q)$ is independent of $\mu$ within errors, as one would expect. For $p_{\mu} \neq 0$, the $F_{4}$ form factor appears, and this has a large effect

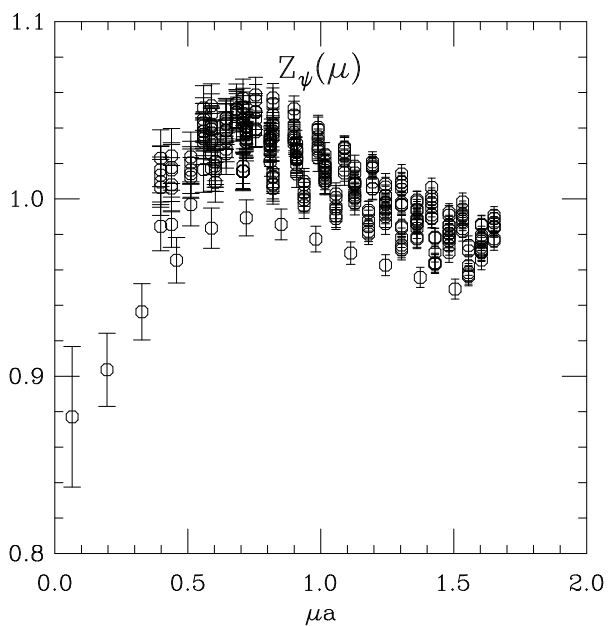

Figure 1. $Z_{\psi}(\mu)$ as a function of $\mu$, for 80 configurations.

on $K_{\mu}$ - making it consistent with 0 for all but the smallest values of $p_{\mu}$.

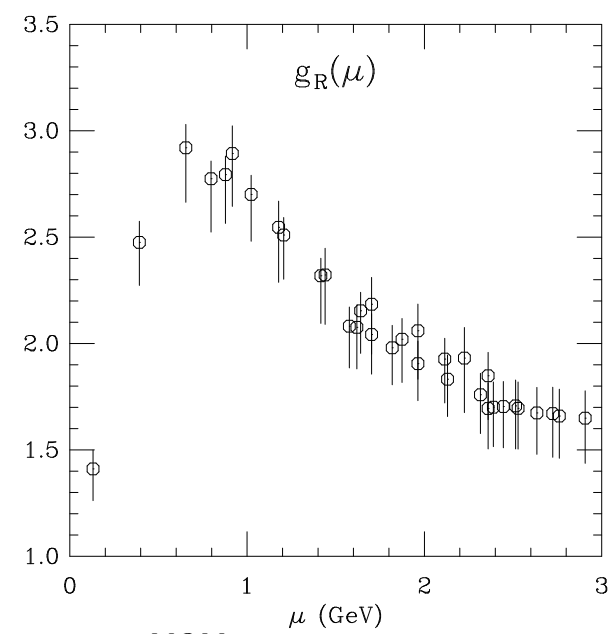

Figure 2. $g_{R}^{M O M}(\mu)$ as a function of $\mu$.

The renormalised coupling is showed as a function of $\mu$ in figure 2. The signal is encouragingly clean, and it exhibits qualitatively the same behaviour as the running coupling extracted from the 3 -gluon vertex 四. 
An estimate for $\Lambda_{Q C D}$ in this scheme has been obtained by inverting equation (9), and the result is plotted as a function of momentum in figure 3 . The running of $g_{R}^{M O M}$ will be consistent with twoloop continuum perturbation theory where this estimate is consistent with a constant. However, no firm conclusions can be drawn in this case, since the point where the data becomes consistent with a constant value for $\Lambda$ (above $1 \mathrm{GeV}$ ) is also where noise begins to dominate.

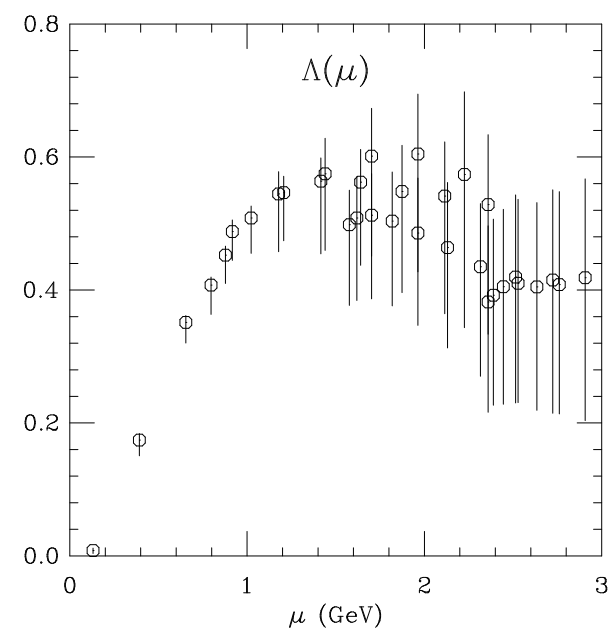

Figure 3. $\Lambda^{M O M}$ as a function of the renormalisation scale $\mu$.

\section{Discussion and conclusions}

The feasibility of using the quark-gluon vertex to extract the QCD running coupling has been demonstrated, although $O(a)$ errors in the quark propagator remain a problem. This may be addressed by using an off-shell improved fermion action, but further work is needed to investigate the feasibility of this.

These results will be matched perturbatively to the $\overline{M S}$ scheme to enable a quantitative comparison with other determinations of $\alpha_{s}$. A study of the other form factors in the vertex, as well as the possible effects of Gribov copies, is also underway.

\section{Acknowledgements}

This work has been supported by Norwegian Research Council grant 100229/432, the Australian Research Council, and EPSRC grants GR/K41663 and GR/K55745. I wish to thank Claudio Parrinello, David Richards and Tony Williams for fruitful discussions.

\section{REFERENCES}

1. G. Martinelli, C. Pittori, C. T. Sachrajda, M. Testa and A. Vladikas, Nucl. Phys. B 445 81 (1995)

2. J. I. Skullerud, Nucl. Phys. B 47 (Proc. suppl.), 398 (1996)

3. S. Sharpe, these proceedings

4. C. Parrinello, Phys. Rev. D 50, 4247 (1994); B. Allés et al., hep-lat/9606033 and these proceedings. 